Artículo

\title{
Variaciones en las respuestas termoregulatorias de ovejas de pelo durante los meses de verano en un clima desértico
}

Variations in the thermoregulatory responses of hair ewes during the summer months in a desert climate

Ulises Macías-Cruz ${ }^{\mathrm{a}^{*}}$

Miguel A. Gastélum ${ }^{\mathrm{a}}$

Leonel Avendaño-Reyes ${ }^{\mathrm{a}}$

Abelardo Correa-Calderón ${ }^{\mathrm{a}}$

Miguel Melladob

Alfonso Chay-Canul ${ }^{\mathrm{c}}$

Carlos F. Arechiga ${ }^{\mathrm{d}}$

a Universidad Autónoma de Baja California, Instituto de Ciencias Agrícolas, Mexicali, Baja California, México.

${ }^{\mathrm{b}}$ Universidad Autónoma Agraria Antonio Narro, Departamento de Nutrición Animal, Saltillo, Coahuila, México.

c Universidad Juárez Autónoma de Tabasco, División Académica de Ciencias Agropecuarias, Villahermosa, Tabasco, México.

${ }^{\mathrm{d}}$ Universidad Autónoma de Zacatecas, Unidad Académica de Medicina Veterinaria y Zootecnia, Zacatecas, México.

*Autor de correspondencia: ulisesmacias1988@ hotmail.com

\section{- Resumen:}

Se confinaron 10 ovejas multíparas Katahdin $\times$ Pelibuey, color blanco, no gestantes y no lactantes, para evaluar las variaciones diurnas de variables fisiológicas y concentración 
de algunos metabolitos sanguíneos a través de los meses calientes de verano (junioseptiembre), en una región desértica del noroeste de México. Las concentraciones de metabolitos se midieron a las 6 y $18 \mathrm{~h}$ y las variables fisiológicas a las $0,6,12$ y $18 \mathrm{~h}$ durante cuatro días de cada mes (mediciones semanales). Los promedios para temperatura ambiental e índice de temperatura-humedad durante el verano fueron $33.6{ }^{\circ} \mathrm{C}$ y 78.6 unidades, respectivamente, siendo agosto el mes más caliente. No cambió $(P>0.05)$ el peso vivo y la condición corporal a través de los meses de verano. La temperatura rectal en agosto fue menor $(P<0.05)$ entre 0 y $12 \mathrm{~h}$, y mayor $(P<0.05)$ a las $18 \mathrm{~h}$ comparado con los otros meses. La frecuencia respiratoria descendió $(P<0.05)$ a las $6 \mathrm{~h}$ en todos los meses, pero a las 0 y $18 \mathrm{~h}$ fue mayor $(P<0.05)$ en agosto que en los otros meses. Se observaron mayores $(P<0.05)$ concentraciones de glucosa y menores $(P<0.05)$ concentraciones de colesterol y triglicéridos en junio y julio con relación a agosto y septiembre. Se concluye que las ovejas de pelo mantienen homeotermia durante los meses de verano en regiones desérticas por cambiar el ritmo diurno de sus variables fisiológicas y metabolitos, de acuerdo con la intensidad del estrés calórico de cada mes.

- Palabras clave: Ovinos de pelo, Estrés calórico, Homotermia, Adaptación fisiológica.

\section{- Abstract:}

Ten (10) multiparous hair ewes from white genotype, non-pregnant and non-lactating, were confined in order to determine diurnal variations of physiological variables and some blood metabolite concentrations through hot summer months (June to September) in an desert region of the northwest Mexico. While blood metabolite concentrations were measured at 6 and $18 \mathrm{~h}$, physiological variables were evaluated at $0,6,12$ and $18 \mathrm{~h}$ during 4-d in each month (weekly measurements). Temperature and temperature-humidity index monthly averages during the study were $33.6{ }^{\circ} \mathrm{C}$ and 78.6 units, respectively, being August the hottest month. Live weight and body condition did not change $(P>0.05)$ across the summer months. Compared with other months, rectal temperatures in August were lower $(P<0.05)$ between 0 and $12 \mathrm{~h}$, and then increased $(P<0.05)$ at $18 \mathrm{~h}$. Respiration frequency decreased $(P<0.05)$ at $6 \mathrm{~h}$ in all the months, but at 0 and $18 \mathrm{~h}$ this was higher in August than in other months. Higher $(P<0.05)$ glucose concentration and lower $(P<0.05)$ cholesterol and triglyceride concentration were observed in June and July than in August and September. In conclusion, under desert climatic conditions, hair ewes had the ability to maintain homeothermy during all summer warm months because they changed their diurnal rhythms of physiological and metabolic variables according to the intensity of the heat stress in each month.

- Key words: Hair sheep, Heat stress, Homeothermy, Physiological adaptation.

Recibido: 13/06/2017

Aceptado: 19/01/2018 


\section{| Introducción V}

La mayor parte del noroeste de México se caracteriza por ser una región árida, con un clima muy seco, donde la presencia de lluvias es escasa y los veranos son muy calientes ${ }^{(1)}$. Las temperaturas que se registran en esta región llegan a ser superiores a $50{ }^{\circ} \mathrm{C}$ en el verano, por lo cual se considera que los animales domésticos se encuentran en un ambiente de estrés por calor durante los meses de esta época ${ }^{(2)}$. Las elevadas temperaturas de verano tienen un impacto negativo en la capacidad productiva y reproductiva de los ovinos, ya que su prioridad es activar mecanismos de termorregulación para mantener la homotermia $^{(3)}$.

Los ovinos de raza de pelo como Pelibuey, Dorper, Katahdin o sus cruzas tienen una alta capacidad para adaptarse a las condiciones climáticas del noroeste de México, de tal manera que durante el verano estas razas han mostrado la capacidad de termorregular su cuerpo $^{(4)}$ sin comprometer drásticamente la actividad reproductiva ${ }^{(5)}$ y la ganancia de peso en la finalización ${ }^{(6)}$. Algunos estudios han elucidado que los ovinos de pelo pueden adaptarse fácilmente a condiciones de estrés térmico porque, a diferencias de las razas de lana, reducen la producción de calor metabólico ${ }^{(7)}$, respiran más lento y profundo $^{(8)}$, y las células mononucleares presentan más viabilidad por tener mayores concentraciones de la proteína del choque calórico HSP $70^{(9)}$. Adicionalmente, un estudio reciente indicó que las ovejas de pelo mantienen condiciones de normotermia durante los veranos cálidos del noroeste de México, debido a la activación de mecanismos adaptativos fisiológicos, los cuales favorecen la pérdida de calor corporal a través de la piel durante las horas menos calientes del día (vías no evaporativas), e incrementan drásticamente la frecuencia respiratoria (FR; vía evaporativa) cuando el gradiente de temperatura rectal (TR) y ambiental es pequeño ${ }^{(10)}$.

Cabe mencionar que gran parte del conocimiento generado para comprender cómo los ovinos de pelo se adaptan al estrés térmico, se ha desarrollado usando cámaras termoambientales y aplicando estrés térmico de tipo agudo. Sin embargo, bajo condiciones naturales de verano en las regiones áridas, el estrés calórico al que son expuestos los animales es crónico e intenso, con variaciones en las temperaturas ambientales de acuerdo con la hora del día y mes. Así, en junio y septiembre se presentan temperaturas ambientales que generan condiciones de estrés calórico menos intensas a las observadas en julio y agosto ${ }^{(5)}$. Estas variaciones en las temperaturas diurnas y mensuales a través del verano, pueden llevar a ciertos ajustes fisiológicos y metabólicos específicos que permitan un uso más eficiente de la energía metabolizable consumida ${ }^{(3)}$. Por lo tanto, el objetivo de este estudio fue determinar la variación en las constantes fisiológicas y en 
algunos metabolitos sanguíneos a través de los meses calientes del verano en ovejas de pelo mantenidas bajo condiciones áridas del noroeste de México.

\section{Material y métodos V}

El estudio se condujo durante el verano de 2013 en la Unidad Experimental Ovina del Instituto de Ciencias Agrícolas, de la Universidad Autónoma de Baja California (UABC), localizada en el Valle de Mexicali, Baja California, México ( $32^{\circ} 24^{\prime} \mathrm{LN}$ y $115^{\circ} 22^{\prime} \mathrm{LO}$ ). La región se caracteriza por tener un clima desértico cálido (Bwh), con temperaturas extremas en verano $\left(40.5^{\circ} \mathrm{C}\right)$ e invierno $\left(7.4^{\circ} \mathrm{C}\right)$, y escasa precipitación pluvial, la cual se concentra principalmente en diciembre $(85 \mathrm{~mm} \text { anuales })^{(1)}$.

\section{- Animales y manejo •}

Se utilizaron 10 ovejas multíparas Katahdin $\times$ Pelibuey, color blanco, no gestantes y no lactantes, con un peso vivo (PV) inicial de $53.5 \pm 4.1 \mathrm{~kg}$ y una condición corporal (CC) de $3.2 \pm 0.2$ unidades (escala 1-5). En el mes de mayo, un mes antes de iniciar las mediciones experimentales, las ovejas fueron vitaminadas y desparasitadas, así como adaptadas a convivir en un mismo corral $(5 \times 6 \mathrm{~m})$, el cual estaba equipado con comederos en el centro, bebedero y sombra $\left(1.2 \mathrm{~m}^{2} /\right.$ animal $)$ de lámina galvanizada a una altura de $2.5 \mathrm{~m}$. Las paredes de los corrales estaban construidas de malla de acero para facilitar el flujo de aire. La alimentación antes y durante el periodo experimental consistió de una dieta de mantenimiento, que contenía paja de trigo y heno de alfalfa picado en una proporción 50:50. Tanto la dieta como el agua limpia se ofrecieron ad libitum a las 0700 y 1700 h todos los días. La composición de la dieta fue $90 \%$ de materia seca, $10 \%$ de proteína cruda y 1.8 Mcal de energía metabolizable/kg de materia seca. 


\section{- Tratamientos y mediciones •}

Los tratamientos consistieron en cada uno de los meses de verano (junio, julio, agosto y septiembre), en los cuales se hicieron mediciones de PV y CC, así como de variables climáticas, fisiológicas y metabólicas. Una vez por mes (día 14) se registró individualmente PV y CC (escala de 5 puntos) ${ }^{(11)}$. Las variables climáticas se midieron diariamente, mientras que las variables fisiológicas y metabólicas se evaluaron a intervalos siete días en cada mes (cuatro días de muestreo/mes). Las variables climáticas y fisiológicas se determinaron en horarios de 0000 - 0100 h (medianoche), 0600 - $0700 \mathrm{~h}$ (mañana), 1200 - 1300 h (mediodía) y 1800 - 1900 h (tarde), mientras que las metabólicas se midieron solamente en la mañana y la tarde de acuerdo con los horarios previos.

Las variables climáticas como temperatura $\left(\mathrm{T},{ }^{\circ} \mathrm{C}\right)$ y humedad relativa $(\mathrm{HR}, \%)$ se obtuvieron de la estación meteorológica universitaria perteneciente a UABC. El índice de temperatura-humedad (ITH) se calculó usando la siguiente fórmula ${ }^{(12)}$ : ITH $=0.81 \times \mathrm{T}+$ $\mathrm{HR}(\mathrm{T}-14.4)+46.4$. Las variables fisiológicas evaluadas fueron TR, FR y temperatura de capa de pelo en diferentes regiones del cuerpo (cabeza, flanco derecho, vientre, paleta y anca). La TR se midió introduciendo por el recto un termómetro digital (Delta Track, $\mathrm{CA} \circledast$, USA), la FR contando el número de respiraciones por minuto (rpm) y la temperatura de capa de pelo tomando fotos con una cámara termográfica (Fluke Ti10, USA) a una distancia de $2.0 \mathrm{~m}$ del cuerpo del animal. Posteriormente, las fotos se descargaron en la computadora para obtener las temperaturas de las áreas corporales especificadas previamente usando el software Fluke SmartView ${ }^{\circledR}$ 3.9.

Adicionalmente, se colectaron muestras sanguíneas de la vena yugular en tubos vacutainer de $10 \mathrm{ml}$, las cuales se centrifugaron a 3,500 xg durante $15 \mathrm{~min}$, a una temperatura de $10^{\circ} \mathrm{C}$. Después se separó el suero en viales de $2 \mathrm{ml}$ y se almacenó a $-20^{\circ} \mathrm{C}$ hasta la determinación de metabolitos séricos (glucosa, colesterol y triglicéridos) con un analizador automático de química sanguínea (Modelo DT-60, Johnson \& Johnson, USA).

\section{- Análisis estadístico •}

Toda la información se sometió a análisis de varianza usando el procedimiento MIXED y se hizo la comparación de medias usando la opción PDIFF del programa $\mathrm{SAS}^{(13)}$. Se utilizó un diseño completamente al azar (DCA) considerando cada mes como tratamiento para PV y CC. En las variables fisiológicas y metabólicas se utilizó un DCA con mediciones repetidas en el tiempo, donde mes, hora del día y la interacción mes $\times$ hora 
del día se consideraron como efectos fijos. Se indicaron diferencias estadísticas cuando $P<0.05$.

\section{$\|$ Resultados $\|$}

Los resultados de las condiciones climáticas se encuentran en el Cuadro 1. Las variables climáticas presentaron una variación diurna similar en todos los meses de verano. Las medias de T e ITH fueron bajas a medianoche $\left(31^{\circ} \mathrm{C}\right.$ y 76 unidades $)$ y en las mañanas ( $27^{\circ} \mathrm{C}$ y 74 unidades), y aumentaron a mediodía ( $37^{\circ} \mathrm{C}$ y 82 unidades) y en las tardes (39 ${ }^{\circ} \mathrm{C}$ y 82 unidades). Los meses de julio y agosto fueron los más calientes, pero septiembre fue el mes más húmedo; en consecuencia, los ITH de esos tres meses se ubicaron entre 78.4 y 80.4 unidades. El ITH en junio fue ligeramente menor (76.8 unidades) que en los otros meses. Los resultados de PV y CC por efecto de mes se muestran en la Figura 1, y ambas variables de estado corporal no cambiaron $(P>0.05)$ a través de los meses de verano.

Cuadro 1: Condiciones climáticas prevalecientes durante los meses de verano en la región desértica del noroeste de México

\begin{tabular}{lcccc}
\hline & \multicolumn{4}{c}{ Meses de verano } \\
\cline { 2 - 5 } & Junio & Julio & Agosto & Septiembre \\
\hline \multirow{4}{c}{ Temperatura, ${ }^{\circ} \mathrm{C}$} \\
Medianoche & $28.85 \pm 1.06$ & $32.98 \pm 1.05$ & $33.07 \pm 0.97$ & $29.30 \pm 0.80$ \\
Mañana & $26.23 \pm 1.46$ & $27.60 \pm 0.68$ & $27.77 \pm 0.29$ & $26.65 \pm 0.31$ \\
Mediodía & $36.55 \pm 1.22$ & $38.53 \pm 1.14$ & $39.58 \pm 1.33$ & $34.00 \pm 1.02$ \\
Tarde & $38.94 \pm 1.45$ & $40.58 \pm 1.11$ & $41.45 \pm 0.99$ & $36.38 \pm 1.11$ \\
Promedios & $32.64 \pm 5.12$ & $34.92 \pm 4.20$ & $35.50 \pm 4.43$ & $31.58 \pm 3.97$ \\
\multicolumn{4}{c}{ Humedad relativa, $\%$} \\
Medianoche & $15.64 \pm 1.79$ & $31.30 \pm 2.41$ & $23.55 \pm 3.53$ & $48.00 \pm 1.79$ \\
Mañana & $39.02 \pm 1.46$ & $37.51 \pm 1.59$ & $63.08 \pm 1.67$ & $65.53 \pm 1.00$ \\
Mediodía & $17.05 \pm 2.08$ & $20.54 \pm 3.60$ & $25.02 \pm 2.85$ & $39.17 \pm 3.14$ \\
Tarde & $12.24 \pm 0.65$ & $15.49 \pm 1.06$ & $16.57 \pm 0.68$ & $25.15 \pm 1.47$ \\
Promedios & $20.99 \pm 8.03$ & $26.21 \pm 10.61$ & $32.05 \pm 11.84$ & $44.46 \pm 10.00$ \\
& Índice de temperatura-humedad, unidades & \\
Medianoche & $73.99 \pm 0.84$ & $75.69 \pm 0.73$ & $77.25 \pm 0.44$ & $76.97 \pm 0.64$ \\
Mañana & $71.81 \pm 0.77$ & $73.62 \pm 1.05$ & $77.05 \pm 0.17$ & $75.74 \pm 0.38$ \\
Mediodía & $80.15 \pm 0.72$ & $82.32 \pm 0.32$ & $83.29 \pm 0.70$ & $81.28 \pm 0.40$ \\
Tarde & $81.34 \pm 1.20$ & $82.09 \pm 0.86$ & $84.03 \pm 0.79$ & $81.01 \pm 0.82$ \\
Promedios & $76.82 \pm 3.87$ & $78.43 \pm 2.92$ & $80.40 \pm 2.48$ & $78.75 \pm 2.23$ \\
\hline
\end{tabular}


Figura 1: Cambios de peso vivo (PV) y condición corporal (CC) a través de los meses de verano en ovejas de pelo $(P<0.05)$

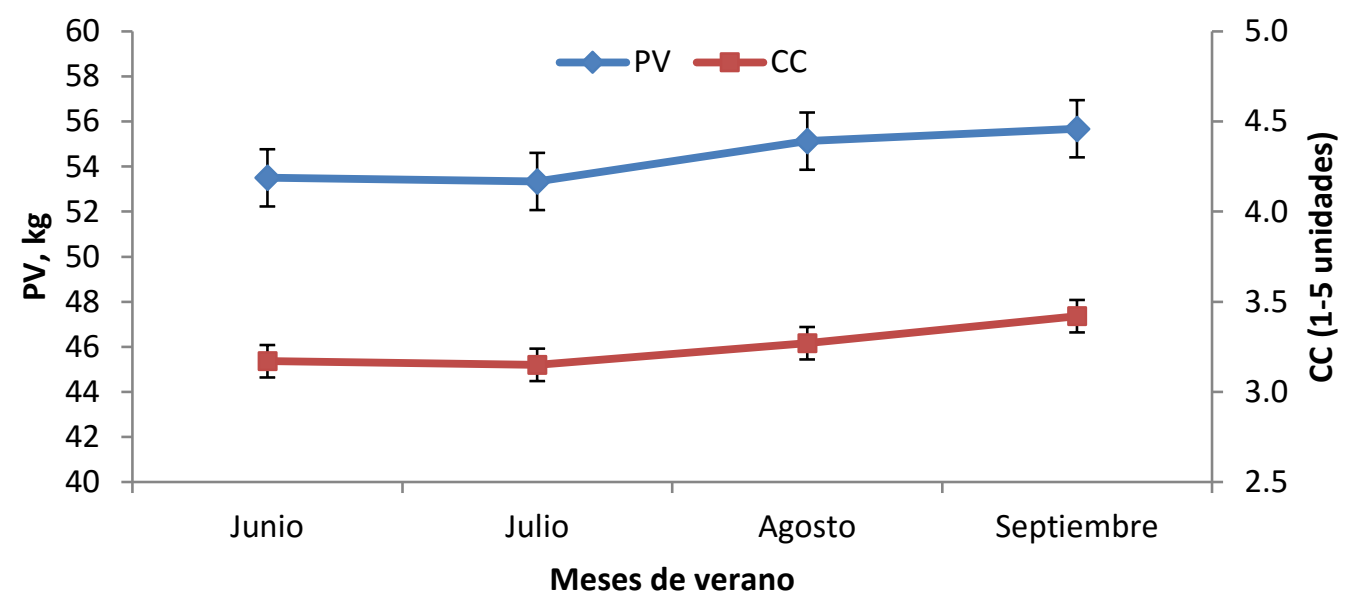

Los resultados de TR y FR por efecto de la interacción mes x hora del día se presentan en la Figura 2. Las TR y FR cambiaron $(P<0.05)$ principalmente en agosto con relación a los otros meses de verano. Comparado con los otros meses, la TR disminuyó $(P<0.05)$ en agosto desde medianoche hasta mediodía y aumentó $(P<0.05)$ por la tarde. La FR permaneció alta $(P<0.05)$ en agosto desde la tarde hasta medianoche y disminuyó $(P<0.05)$ a mediodía sin diferencias en la mañana con relación a los otros meses.

Figura 2: Temperatura rectal (TR) y frecuencia respiratoria (FR) durante los meses de verano en ovejas de pelo $(* P<0.05$ indica diferencia entre meses en cada hora del día)
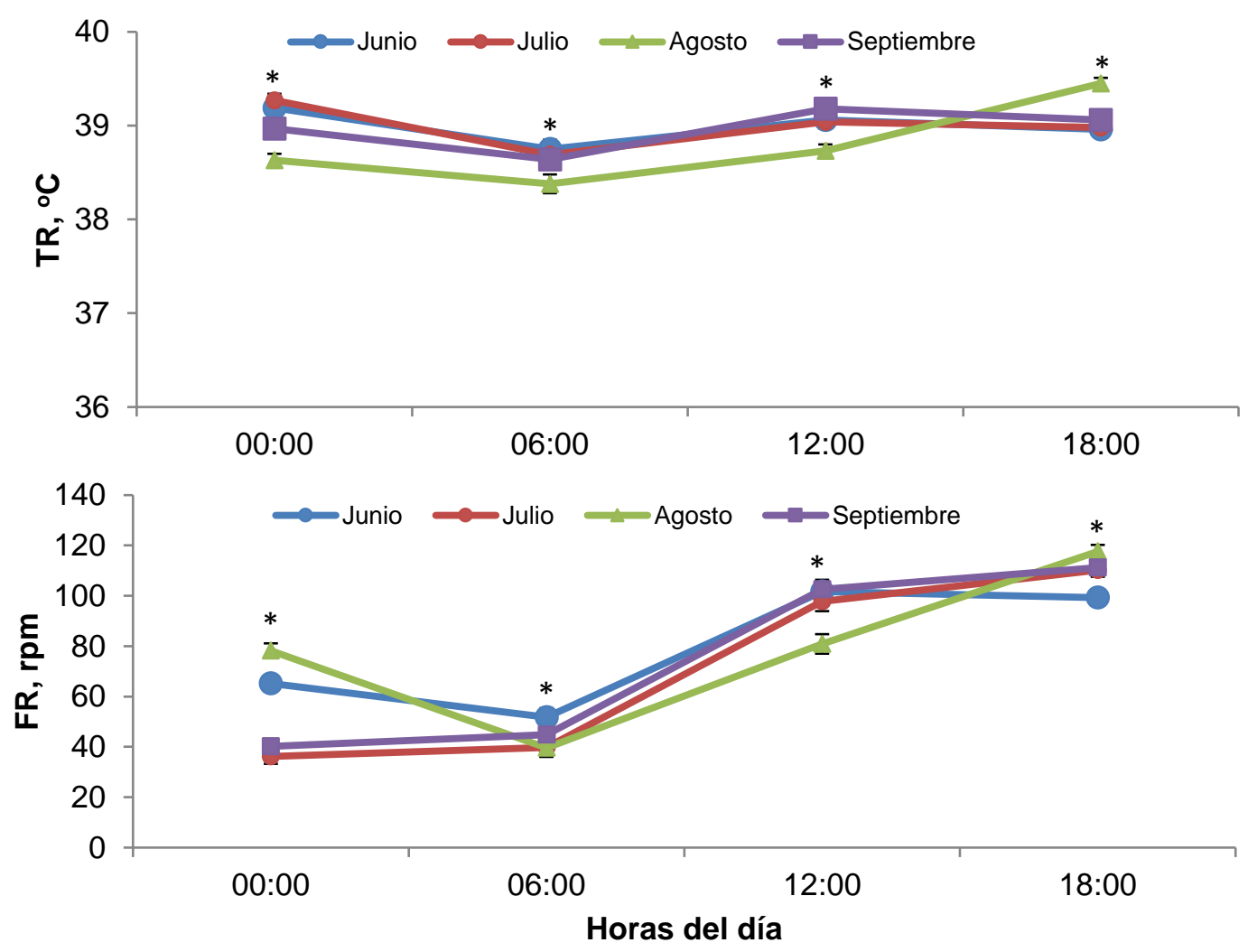
Los resultados de temperatura en la capa de pelo de las diferentes regiones corporales evaluadas se presentan en las Figuras 3 y 4. Las temperaturas de las diferentes áreas corporales fueron más altas $(P<0.05)$ a mediodía y más bajas $(P<0.05)$ entre medianoche y las mañanas en todos los meses de verano. Sin embargo, la temperatura de la cabeza no se afectó $(P>0.05)$ a medianoche ni en la mañana en ninguno de los meses de evaluados, pero las temperaturas del flanco derecho, paleta y anca fueron más elevadas $(P<0.05)$ a medianoche en agosto, así como en las mañanas en los meses de junio y agosto, en comparación con los otros meses. A mediodía las temperaturas de todas las regiones corporales fueron mayores $(P<0.05)$ en septiembre y menores $(P<0.05)$ en julio, pero en la tarde fueron mayores $(P<0.05)$ en agosto y menores $(P<0.05)$ en septiembre.

Figura 3: Temperatura de la cabeza (Tcab) y del flanco derecho (TFD) durante los meses de verano en ovejas de pelo $(* P<0.05$ indica diferencia entre meses en cada hora del día)
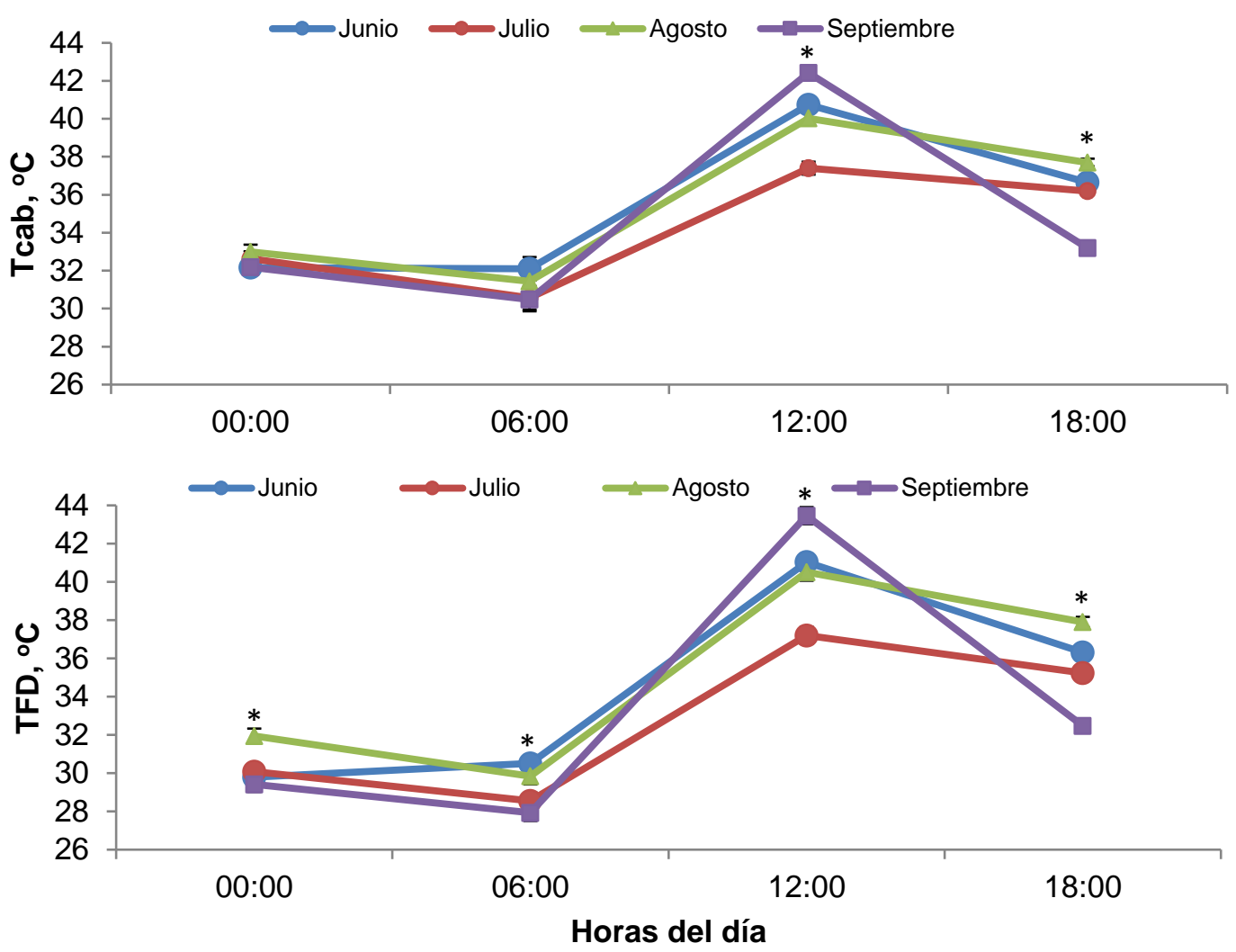
Figura 4: Temperatura de la paleta (Tpal) y el anca (Tanca) durante los meses de verano en ovejas de pelo $\left({ }^{*} P<0.05\right.$ indica diferencia entre meses en cada hora del día)
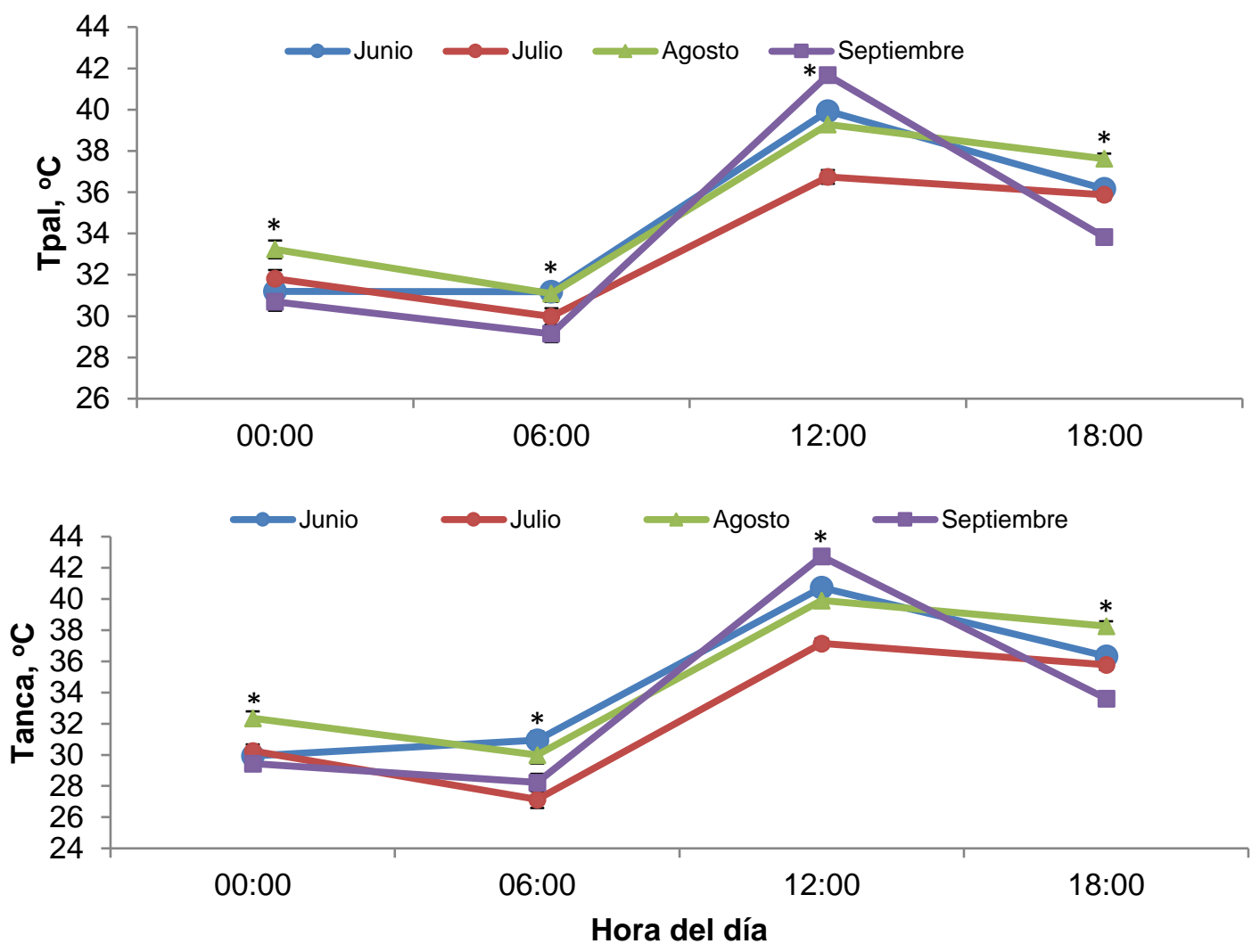

Los resultados de concentraciones de metabolitos séricos se muestran en el Cuadro 2. La interacción entre mes y hora del día no afectó $(P>0.05)$ la concentración de los metabolitos. Los niveles de glucosa fueron mayores $(P<0.05)$ en junio y julio que en agosto y septiembre, aunque resultados contrarios $(P<0.05)$ fueron observados para las concentraciones de colesterol y triglicéridos entre estos meses. Por otra parte, las concentraciones de todos los metabolitos fueron menores $(P<0.05)$ en las mañanas que en las tardes.

Cuadro 2: Variaciones en las concentraciones de metabolitos en suero a través de los meses de verano en ovejas de pelo $\left(\mathrm{mg} \mathrm{dl}^{-1}\right)$

\begin{tabular}{llll}
\hline Variables & Glucosa & Colesterol & Triglicéridos \\
\hline Meses: & & & \\
Junio & $60.90 \pm 1.09^{\mathrm{a}}$ & $70.65 \pm 3.38^{\mathrm{a}}$ & $30.33 \pm 2.20^{\mathrm{a}}$ \\
Julio & $62.12 \pm 1.09^{\mathrm{a}}$ & $73.25 \pm 3.38^{\mathrm{a}}$ & $40.05 \pm 2.20^{\mathrm{b}}$ \\
Agosto & $52.95 \pm 1.09^{\mathrm{b}}$ & $85.05 \pm 3.38^{\mathrm{b}}$ & $48.65 \pm 2.20^{\mathrm{c}}$ \\
Septiembre & $54.05 \pm 1.09^{\mathrm{b}}$ & $94.89 \pm 3.38^{\mathrm{b}}$ & $51.00 \pm 2.20^{\mathrm{c}}$ \\
Hora del día: & & & \\
Mañana & $54.90 \pm 0.78^{\mathrm{a}}$ & $76.67 \pm 2.40^{\mathrm{a}}$ & $38.90 \pm 1.57^{\mathrm{a}}$ \\
Tarde & $57.11 \pm 0.78^{\mathrm{b}}$ & $85.25 \pm 2.40^{\mathrm{b}}$ & $46.12 \pm 1.57^{\mathrm{b}}$ \\
\hline
\end{tabular}

${ }^{\text {ab }}$ Superíndices diferentes dentro de columna y factor de estudio indican diferencias $(P<0.05)$. 


\section{" Discusión V}

La zona de confort térmica para los ovinos de pelo se encuentra entre los 15 y $30^{\circ} \mathrm{C}^{(14)}$ y un ITH de <74 unidades es clasificado como ausencia de estrés calórico en dichos ovinos $^{(15)}$. En este estudio, el promedio mensual de la T y el ITH en los meses de verano varió de 31.6 a $35.5^{\circ} \mathrm{C}$ y de 76.8 a 80.4 unidades, respectivamente, lo cual demuestra que las ovejas estuvieron de manera general en condiciones de estrés calórico. Cabe mencionar que la $\mathrm{T}$ en las mañanas fue $<30{ }^{\circ} \mathrm{C}$ en todos los meses, situación que pudo favorecer el alivio temporal diario de las ovejas en las condiciones de hipertermia ${ }^{(15)}$. El tipo de estrés calórico que prevaleció durante el estudio varió de moderado (junio, julio y septiembre) a severo (agosto) ${ }^{(16,17)}$. En las tardes de agosto, el estrés calórico llegó a ser de tipo emergente ( $\geq 84$ unidades).

Las condiciones ambientales de estrés calórico promueven ajustes fisiológicos en los ovinos para disipar la carga de calor corporal $^{(3)}$. En el noroeste de México, se han reportado incrementos en la TR, FR y consumo de agua, así como una ligera disminución en el consumo de alimento y estado corporal por efecto de las condiciones de estrés calórico de verano en ovinos de raza de pelo ${ }^{(4,6,10)}$. También en otros estudios se han observado cambios en el ritmo circadiano de las variables fisiológicas en ovinos adaptados que fueron sometidos a estrés calórico crónico de larga duración ${ }^{(18)}$, tal como sucedió en este trabajo. Las variaciones circadianas en las constantes fisiológicas, a través del día, son consideradas como mecanismos adaptativos que desarrollan algunos animales para ser más eficientes en el uso de la energía de mantenimiento, así como para disipar la carga de calor corporal y evitar la deshidratación ${ }^{(19)}$. En el presente estudio, se detectaron variaciones en la TR, FR y temperaturas de capa de pelo en todos los meses de verano, de acuerdo con los cambios en la T y el ITH observados a través del día. Estos hallazgos coinciden con otras investigaciones donde las variables fisiológicas aumentaron con el incremento en la $\mathrm{T}$ a través del día, tanto bajo condiciones de estrés calórico como de termoneutralidad ${ }^{(10,15,17)}$.

En agosto, que fue el mes más caliente en todos los horarios de muestreo, los valores medios de las constantes fisiológicas difirieron en algunos horarios con respecto a los observados en los otros meses. Comparado con el resto de los meses, en agosto se observó $20 \%$ menos FR a mediodía, pero en la tarde y a medianoche aumentó en 10 y $66 \%$ (ausencia de radiación solar), respectivamente. Esto puede ser un mecanismo de adaptación que presentan las ovejas de pelo para reducir las pérdidas de agua corporal y evitar la deshidratación en condiciones de estrés calórico intenso. El aumento en la FR implica la evaporación de una elevada cantidad de agua, y si la FR aumenta de manera 
desmedida en horas de alta radiación solar, la tasa de evaporación de agua corporal es más elevada que cuando el aumento se da en horas de menor radiación solar ${ }^{(19)}$. Cabe mencionar que el patrón de variación de la temperatura corporal interna en agosto (basado en resultados de TR) fue un factor importante para que se presentara el mecanismo adaptativo mencionado. La menor temperatura interna de 0 a $12 \mathrm{~h}$ en agosto comparado con los otros meses en los mismos horarios, pudo favorecer que las ovejas toleraran una mayor carga de calor corporal con un menor aumento en la FR a mediodía del mes de agosto, justamente en las horas del día con mayor radiación solar. Un estudio previo en ovinos de pelo ya había sugerido la presencia de dicho mecanismo ${ }^{(10)}$, por lo cual estos resultados lo confirman. En animales ungulados adaptados al calor extremo de las regiones desérticas ${ }^{(19,20)}$, así como en ovejas de lana de la raza Merino Australiano ${ }^{(21)}$ y en cabritos ${ }^{(22)}$ mantenidos en estrés calórico crónico, también ya se había observado dicho mecanismo.

El intercambio de calor entre el cuerpo y el ambiente a través de la piel, también juega un papel importante en la termorregulación de ovinos de pelo estresados por calor ${ }^{(3,10)}$. Una temperatura de la piel mayor a la ambiental indica pérdidas de calor corporal, pero cuando es menor la temperatura de la piel se asume que hay una ganancia de calor ambiental ${ }^{(3)}$. En este estudio, se observaron predominantemente en agosto, mayores temperaturas en la paleta, anca y flanco derecho en los horarios de medianoche, mañana y tarde con relación a los otros meses; sin embargo, solamente en las mañanas de agosto hubo disipación de calor corporal a través de la piel en las distintas regiones corporales; en la tarde hubo ganancia de calor corporal y a medianoche se presentó un equilibrio entre la temperatura corporal y la ambiental. Cabe mencionar que julio fue el segundo mes más caliente, pero las temperaturas del pelo no mostraron incrementos tan marcados a través de los horarios como se observó en agosto. No obstante, cuando se compararon las temperaturas del pelo con las ambientales en cada horario dentro del mes de julio, se evidenció que las ovejas perdieron calor solamente por las mañanas en la cabeza y paleta, $\mathrm{y}$ en los otros horarios mostraron estar principalmente ganando calor ambiental por las diferentes regiones corporales. Las temperaturas de la capa de pelo mostraron en junio y septiembre que las pérdidas de calor corporal se presentan de medianoche a mediodía, pero no por las tardes. En general, los resultados de termografía infrarroja evidenciaron que las pérdidas de calor corporal a través de la piel, son efectivas en ovinos de pelo principalmente en los meses menos calientes de verano en regiones desérticas del noroeste de México.

Comparado con los otros meses, se esperaba que la menor TR observada en agosto de 0 a $12 \mathrm{~h}$, fuera explicada por un aumento en las pérdidas de calor corporal a través de la piel en horas de la noche y la mañana, tal como se reportó en un estudio previo realizado en el mismo sitio experimental durante el verano ${ }^{(10)}$. Sin embargo no fue así, dado que solo en las mañanas de agosto hubo disipación de carga de calor corporal. En consecuencia, esto sugiere que junto con el aumento de la FR, posiblemente en agosto hubo mecanismos metabólicos que ayudaron a reducir la temperatura corporal durante las horas de la noche y la mañana. Es posible que una reducción en la producción de calor 
metabólico sea parcialmente responsable de los resultados de la TR de agosto. Algunos estudios han encontrado que las concentraciones de hormonas tiroideas disminuyen por efecto del estrés calórico cuando los ajustes fisiológicos son incapaces de mantener homeotermia en rumiantes ${ }^{(3,7)}$.

El metabolismo energético en las ovejas de pelo varió a través de los meses de verano, posiblemente como una respuesta a la acumulación de calor corporal. Los efectos del estrés calórico sobre las concentraciones de metabolitos en ovinos son variados, y muchos factores tanto inherentes al animal, como relacionados con la alimentación y el tipo de estrés calórico, podrían estar influyendo. En este estudio se determinó que permanecieron altos los niveles de glucosa en los primeros dos meses de verano y después disminuyeron en los otros dos meses finales, sin que esta caída en las concentraciones de glucosa fuera considerada drástica ( $\sim 9$ \%). Esto coincide con otros estudios donde reportaron que la concentraciones sanguíneas de glucosa decrecen a un pequeño nivel por efecto del estrés calórico en ovinos, independientemente del consumo de alimento ${ }^{(23,24,25)}$. El hecho de que los niveles de glucosa no caigan súbitamente se debe a que dicho metabolito es una fuente de energía regulada homeostáticamente ${ }^{(24)}$. Posiblemente, la glucosa sérica fue menor en agosto y septiembre debido a una disminución en las reservas de glucógeno en hígado, producto del desgaste energético que implica mantener la tasa respiratoria alta durante periodos prolongados ${ }^{(10)}$. El incremento de la FR es el ajuste fisiológico que mayor gasto de energía tiene, lo cual es debido a la gran cantidad de músculos que se contraen dentro $\mathrm{y}$ alrededor del tracto respiratorio ${ }^{(25)}$. Otra causa puede ser el aumento en los niveles séricos de insulina debidos al estrés calórico crónico, ya que la insulina favorece la entrada de mayor cantidad de glucosa a las células, promoviendo su disminución en la circulación sanguínea ${ }^{(26)}$.

Las variaciones en las concentraciones séricas de colesterol y triglicéridos mostraron ser inversas a las de glucosa a través de los meses de verano. Esto sugiere que, en ovinos de pelo, el metabolismo lipídico se activa en condiciones de estrés calórico crónico de larga duración como un posible mecanismo para compensar la disminución en la disponibilidad de glucosa, o bien, es un mecanismo alterno para promover el ahorro de glucosa en condiciones de hipertermia crónica. Baumgard y Rhoads ${ }^{(26)}$ mencionan que condiciones de estrés calórico en rumiantes pueden promover la presencia de mecanismos ahorradores de glucosa, siendo el único mecanismo conocido hasta ahora el aumento de lactato sanguíneo para ser usado como fuente de energía en tejidos capaces de oxidarlo. Por otra parte, se desconoce el origen del colesterol y triglicéridos que promovió el aumento de estos metabolitos en el suero en los meses de agosto y septiembre para las ovejas del estudio. La lipolisis del tejido graso no es el origen de estos metabolitos lipídicos porque PV y CC de las ovejas se mantuvo constante a través de los meses de verano. De hecho, numéricamente, el PV aumentó en promedio $1.7 \mathrm{~kg}$ y la CC 0.2 unidades en los meses de agosto y septiembre comparados con los meses de junio y julio. Probablemente, el exceso de colesterol y triglicéridos en la segunda mitad del verano tengan su origen en la síntesis endógena del hígado como consecuencia de un aumento en los niveles sanguíneos de insulina. Se ha reportado que la insulina activa genes lipogénicos a nivel del hígado, 
principalmente aquellos que codifican con enzimas ligadas a la biosíntesis de colesterol $^{(27)}$. Si bien, la insulina no fue medida en este estudio, los resultados de PV y $\mathrm{CC}$ sugieren que las ovejas de pelo tuvieron altos niveles de esta hormona. Estudios previos han demostrado que un aumento en los niveles de insulina sanguínea favorece la ganancia de masa corporal y evita la movilización de reservas corporales en ovinos estresados por calor, ya que dicha hormona metabólica detiene la lipólisis y estimula la lipogénesis ${ }^{(24,26)}$.

Por otra parte, las concentraciones de los metabolitos séricos mostraron una variación diurna en las ovejas utilizadas, lo que sugiere alteraciones de corto plazo en su metabolismo energético para evitar hipertermia. El aumento en los niveles de glucosa, colesterol y triglicéridos por la tarde, se atribuyó parcialmente al incremento de la FR que se presentó en esa parte del día durante todos los meses de verano ${ }^{(28)}$. También, las fluctuaciones en el consumo de alimento a través del día podrían explicar estas variaciones diurnas en los metabolitos sanguíneos ligados al metabolismo energético ${ }^{(29)}$.

\section{Conclusiones e implicaciones $\|$}

Se concluye que las ovejas de pelo presentaron diferencias en sus constantes fisiológicas a través de los meses del verano bajo condiciones desérticas del noroeste de México, las cuales mostraron ser acordes con las variaciones de la temperatura ambiental. Asimismo, el metabolismo energético se ajustó a los diferentes tipos de estrés calórico que experimentaron las ovejas a través de los meses de verano. Un mecanismo adaptativo fisiológico para evitar la deshidratación fue activado en agosto que fue el mes más cálido. Lo anterior implica que las ovejas de pelo se adaptan bien a la agresión térmica de los veranos cálidos en las regiones desérticas.

\section{N Agradecimientos N}

Este estudio fue financiado en el marco de la convocatoria de apoyo a nuevos PTC, PROMEP 2010. Este trabajo forma parte de la investigación desarrollada por el segundo autor durante sus estudios de doctorado en el ICA-UABC, quien también agradece al CONACYT por la beca de manutención otorgada. 


\section{- Literatura citada}

1. INEGI. Anuario estadístico y geográfico de Baja California. 2014. http://www.datatur.sectur.gob.mx/ITxEF_Docs/BCN_ANUARIO_PDF.pdf. Consultado 6 Jun, 2017.

2. Rzedowski J. Vegetación de México. 1. a ed digital. Comisión Nacional para el Conocimiento y Uso de la Biodiversidad, México; 2006. http://www.biodiversidad.gob.mx/publicaciones/librosDig/pdf/VegetacionMx_Con t.pdf. Consultado 6 Jun, 2017.

3. Marai IFM, El-Darawany AA, Fadiel A, Abdel-Hafez MAM. Physiological traits as affected by heat stress in sheep - A review. Small Ruminant Res 2007;71(1-3):1-12.

4. Macías-Cruz U, Gastélum MA, Álvarez FD, Correa A, Díaz R, Meza-Herrera CA, et al. Effects of summer heat stress on physiological variables, ovulation and progesterone secretion in Pelibuey ewes under natural outdoor conditions in an arid region. Anim Sci J 2016;87(3):354-360.

5. Gastélum-Delgado MA, Avendaño-Reyes L, Álvarez-Valenzuela FD, CorreaCalderón A, Meza-Herrera CA, Mellado M, et al. Comportamiento de estro circanual en ovejas Pelibuey bajo condiciones áridas del Noroeste de México. Rev Mex Cienc Pecu 2015;6(1):109-118.

6. Macías-Cruz U, Avendaño-Reyes L, Álvarez-Valenzuela FD, Torrentera-Olivera NG, Meza-Herrera CA, Mellado M, et al. Crecimiento y características de canal en corderos tratados con clorhidrato de zilpaterol durante primavera y verano. Rev Mex Cienc Pecu 2013;4(1):1-12.

7. Ross TT, Goode L, Linnerud AC. Effects of high ambient temperature on respiration rate, rectal temperature, fetal development and thyroid gland activity in tropical and temperate breeds of sheep. Theriogenology 1985;24(2):259-269.

8. Correa MPC, Cardoso MT, Castanheira M, Landim AV, Dallago BSL, Louvandini $\mathrm{H}$, et al. Heat tolerance in three genetic groups of lambs in central Brazil. Small Ruminant Res 2012;104(1-3):70-77.

9. Romero RD, Montero PA, Montaldo HH, Rodríguez AD, Hernández CJ. Differences in body temperature, cell viability and HSP-70 concentrations between Pelibuey and Suffolk sheep under heat stress. Trop Anim Health Prod 2013;45(8):1691-1696.

10. Macías-Cruz U, López-Baca MA, Vicente R, Mejía A, Álvarez FD, Correa-Calderón A, et al. Effects of seasonal ambient heat stress (spring vs. summer) on physiological 
and metabolic variables in hair sheep located in an arid region. Int $\mathbf{J}$ Biometeorol 2016;60(8):1279-1286.

11. Russel AJF, Doney JM, Gunn RJ. Subjective assessment of body fat in live sheep. J Agric Sci 1969;72(3):451-454.

12. Hahn GL. Dynamic responses of cattle to thermal heat loads. J Dairy Sci 1999;82 (Suppl 2):10-20.

13. SAS, SAS/STAT. User's guide statistics (released 9.2). Inc. Cary, NC, USA; SAS Inst. Inc. 2004.

14. Baêta FC, Souza CF. Ambiência em edificações rurais: conforto animal. 2. ${ }^{\mathrm{a}}$ ed. Viçosa, MG: Universidade Federal de Viçosa; 2010.

15. Seixas L, de Melo CB, Tanure CB, Peripolli V, McManus C. Heat tolerance in Brazilian hair sheep. Asian-Australas J Anim Sci 2017;30(4):593-601.

16. Hahn GL, Gaughan JB, Mader TL, Eigenberg RA. Thermal indices and their applications for livestock environments. Livestock energetics and thermal environment management, (Am Soc Agric Biol Engineers, St. Joseph). 2009.

17. Seixas L, de Melo CB, Menezes AM, Ramos AF, Paludo GR, Peripolli V, Tanure CB, Costa Junior JBG, McManus C. Study on environmental indices and heat tolerance tests in hair sheep. Trop Anim Health Prod 2017:49(5):975-982.

18. Kalyan De, Kumar D, Singh AK, Sahoo A, Naqvi SMK. Seasonal variation of physiological response in ewes of farmers flock under semi-arid tropical environment. Biol Rhythm Res 2013;45(3):397-405.

19. Cain IIIJW, Krausman, PR, Rosenstock S, Turner JC. Mechanisms of thermoregulation and water balance in desert ungulates. Wildlife Soc B 2006;34(3):570-581.

20. Taylor CR, Lyman CP. Heat storage in running antelopes: independence of brain and body temperatures. Am J Physiol 1972;222(1):114-117.

21. Alhidary IA, Shini S, Al Jassim RAM, Gaughan JB. Physiological responses of Australian Merino wethers exposed to high heat load. J Anim Sci 2012;90(1):212220.

22. Al-Tamimi HJ. Thermoregulatory response of goat kids subjected to heat stress. Small Ruminant Res 2007;71(1-3):280-285.

23. Mahjoubi E, Amanlou H, Mirzaei-Alamounti HR, Aghaziarati N, Hossein YM, Noori GR, Yuan K, Baumgard. The effect of cyclical and mild heat stress on productivity and metabolism in Afshari lambs. J Anim Sci 2014;92(3):1007-1014. 
24. Mahjoubi E, Hossein YM, Aghaziarati N, Noori GR, Afsarian O, Baumgard. The effect of cyclical and severe heat stress on growth performance and metabolism in Afshari lambs. J Anim Sci 2015;93(4):1632-1640.

25. Indu S, Sejian V, Naqvi MK. Impact of simulated heat stress on growth, physiological adaptability, blood metabolites and endocrine responses in Malpura ewes under semiarid tropical environment. Anim Prod Sci 2015;55(6):766-776.

26. Baumgard LH, Rhoads RP. Effects of heat stress on postabsorptive metabolism and energetics. Annu Rev Anim Biosci 2013;1(7):17-27.

27. Morigny P, Houssier M, Mouisel E, Langin D. Adipocyte lipolysis and insulin resistance. Biochimie 2016;125:259-266.

28. Marai IFM, El-Darawany AA, Fadiel A, Abdel-Hafez MAM. Reproductive performance traits as affected by heat stress and its alleviation in sheep. Trop Subtrop Agroecosys 2008;8(3):209-234.

29. Shehab-El-Deen MAMM, Fadel MS, Soom AV, Saleh SY, Maes D, Leroy JLMR. Circadian rhythm of metabolic changes associated with summer heat stress in highproducing dairy cattle. Trop Anim Health Prod 2010;42(6):1119-1125. 